\title{
STUDY ON THE INFLUENCE OF CONTACT POINTS, SCENARIOS AND GRAPHICAL REFERENCE ELEMENTS ON THE MOTION ANALYSIS PROCESS, CARRIED OUT USING THE INERTIAL MECHATRONIC SYSTEM MVN ANALYZE
}

\author{
Badea Cristian Radu ${ }^{1}$ \\ ${ }^{1}$ National Institute of Research and Development in Mechatronics and Measurement Technique, Bucharest \\ adresacontact@gmail.com
}

\begin{abstract}
The study aimed to perform motion analysis using Xsens's MVN inertial system, in order to assess the impact of „contact points”, „scenarios” and "graphic references elements, on the mentioned process. The analysis were of a qualitative nature, concentrating on the positioning precision with respect to the MVN Analyze global reference system, the imposed exercises being performed freely (without the limitation that electrical cables are coming along with), by the human subject, with, or without the help of some devices (e.g.: the stepper, the parallel-bars assembly)

This study it's not meant to decide the quality of Xsens MVN system, representing just a beginner finding, while performing simple motion analysis.

The analysis of the images was done by relating the positions of the virtual dummy's body segments to the lines and columns that make up the grid of the virtual reference plane, to the position of virtual reference marker, of the calibration's origin and to the global coordinate system of the MVN software.

The exercises for this analysis were chosen in such a manner so that every one of the four scenarios can be covered and also to make possible the use of contact points at the feet and hand level, both simultaneously and individually on each mentioned skeletal segment: „Push-ups” on the hard floor, „Squats” on the training mattress, „Stairs climbing” using the stepper and „Balance” using the balance board.

The results of the analysis were:

- the contact points, graphical reference elements and scenarios, are very important tools into the primary, visual, evaluation of the motion analysis results. They are not just a visual aid (used for the visual effect), but they really are a valid indicator of the position and interaction between the human subject, under analysis and the environment into which the action is taking place;

- by choosing the wrong scenario, or contact points and/or a inadequate combination between the scenario and the contact point's level, the result of the motion analysis can be greatly altered.
\end{abstract}

Keywords: Inertial Motion Analysis System, Mechatronic, „Contact Points”, „Scenarios” and "Graphic References Elements.

\section{Introduction}

The most important disadvantage while using inertial motion analysis system, is the positional drift (positional error) occurrence. The human body's segments positions and orientation is estimated by means of a sensor to segment calibration procedure, starting from the information captured by the analysis systems MEMS, that are attached to the human subject in question, which then are transfer to a biomechanical (scaled) model of the human body.

This calibration introduces errors that may affect the quality of this type of analysis.
This is why, in order to improve the performance of inertial motion analysis, XSens, one of the top producers in this field, has introduced and implemented into his product, namely MVN Analyze, the notions of "contact points”, „scenarios” and "graphic references elements", that may ensure better results and that may be able to minimize the effort of post-processing. Starting from the above mentioned, the overall objective of this study is to explore the possibilities of analysis provided by the inertial motion analysis system MVN, developed by Xsens and to determine if those improvements are having, in fact, a good influence on the quality of the analysis process results. 


\section{Xsens's MVN Inertial Motion Analysis System Specifications, Related to the Article's Subject}

In the MVN environment, the human subject under analysis, is digitally represented, by a virtual dummy (a digital skeleton), the reference plane of the analyzed motion (represented in real life by the floor, the training mattress, the stepper's pedals, or the balance board support surface) is digitally represented by a virtual reference plane, having a grid like aspect and the position of the calibration's origin (the human subject's right heel, or right hand, depending on the chosen settings) it is represented by a red triangle.

In relation to the notions that are the subject of this article, MVN XSens is providing for the user the possibility of choosing between four specific scenarios, depending on the type of the measurement that will be carried out:

- "Single level” - that should be used on analysis in which the human subject's actions are taking place on a level terrain, supposing that there are no variations in ground's height, like in the case of performing push-ups on a flat and incompressible surface;

- „Multi level” - that should be used on analysis in which the human subject's actions are taking place on different levels, supposing that there are variations in ground's height, like in the case of performing the climbing stairs exercise using a stepper, that can simulate a real stair climbing action;

"Soft floor" - that should be used on analysis in which the human subject's actions are taking place on a compressible surface, like in the case of performing different exercises on a training mattress or soft carpet;

- "No level” - that should be used on analysis in which the human subject's actions are taking place while his pelvis remains at the same Hight, in regards with the ground, like in the case of ice skating.

In the MVN environment, while in the calibration stage, the user has to define the direction of the Xaxis, which is established by asking the human subject under analysis to stand in the "N"-pose, facing de forward axis supplied by the MVN's software interface. The right heel of the human subject under analysis it is considered to be the origin of the $\mathrm{X}$-axis and his forward direction has to coincide with the software's forward direction. It is advised that a mark should be placed on the floor, this way, each and every time the calibration has to be applied, the human subject under analysis, will be able to stand in the imposed manner, always having the right heel, placed on the same spot. This measure can ensure the possibility to obtain the same origin of calibration for each and every session. The MVN
Software is representing the obtained origin of calibration (the "0" point of X-axis and Y-axis), by a red triangle, whose tip indicates the positive direction of the $\mathrm{X}$-axis.

Due to the fact that MVN is an inertial analysis system, it doesn't have an absolute positioning system, over time, the virtual dummy (which is the representation of the human subject under analysis into the provided virtual environment, for visual purposes) will show some positional drift, in terms of absolute position in space, with respect to the origin established during calibration.

Another "graphic reference element" is the "grid" which is the virtual equivalent of the floor on to which the action it's taking place, the "0" point of Zaxis. In the same category of relevant graphic elements, the center of mass and the motion path, can also be also included, these being represented in the virtual world by means of a "yellow sphere" and respectively, by a „spatial curve”. In the MVN software the user can set the Hight of the floor.

One of the most important elements implemented into the MVN environment, it's the notion of "contact point". In order to minimize the positional drift, MVN uses this contact points to simulate the interaction between the human subject, under analysis and the physical objects with which he might come in contact with (e.g.: the contact between his feet and the ground, or the contact between his hand and some props objects). There are several predefined contact points on the virtual dummy, considered to be most likely to get in contact with the surroundings:

- the heel of the foot;

- inside, outside and center ball of the foot;

- bony elevation on the upper part of the tibia;

- eminence on the upper part of the femur;

- left, right and center of the buttock;

- base of the spinal column;

- bottom of sternum;

- T4 vertebra;

- shoulder's acromion;

- elbow's olecranon;

- ball of hand;

- top of head.

The MVN software allow the user to edit those standard contact points, so that he can placed them in the most convenient position on the virtual dummy's body segments, but even so, these custom positioning options are restricted to the geometrical elements represented on the graphic skeleton.

When the situation requires it, the MVN software allow the user to ignore a certain contact point, in this case the MVN's fusion engine will decide what will be the most suitable replacement and on what virtual skeletal element to place it.

These above mentioned aspects are most useful in the situations in which the human subject, under analysis, has to climb stairs and the contact points 
have to be translated from one foot to another, or when he has to hang from a bar, using his hands, while practicing pull-ups, or walking on an inclined plan.

\section{The Objective of the Study}

The study aimed to perform motion analysis using Xsens's MVN inertial system, in order to assess the changes that the above-mentioned notions are bringing. The analysis were of a qualitative nature, concentrating on the positioning precision with respect to the MVN Analyze global reference system, the imposed exercises being performed freely (without the limitation that electrical cables are coming along with), by the human subject, with, or without the help of some devices (e.g.: the stepper, the parallel-bars assembly)

This study it's not meant to decide the quality of Xsens MVN system, representing just a beginner finding, while performing simple motion analysis.

\section{Imposed Conditions, the Chosen Exercises And Elements of Interest in the Analysis}

In order to properly evaluate the impact of "contact points”, „scenarios” and "graphic references elements”, on the Xsens MVN motion analysis inertial system's errors, the following measures were taken:

a) For the analysis, a healthy and emotionally balanced, male subject was chosen, with overaverage neuromuscular control;

b) Each of the individual exercise, within each analysis session, was performed with the human subject positioned in the same place, imposing it to maintain the same distance between the feet / hands, if this measure is not ensured by the way that the devices, that are being used for this purpose, are constructed;

c) The "REPROCESS" function was used, so that the data obtained, before and after its use, were compared with each other, in order to verify the system's ability to correct the positioning errors.

d) In order for the proposed goal to get achieved, all of the four scenarios provided by the MVN Analyze software, were used.

The exercises for this analysis were chosen in such a manner so that every one of the four scenarios can be covered and also to make possible the use of contact points at the feet and hand level, both simultaneously and individually on each mentioned skeletal segment:

- „Push-ups” on the hard floor - was chosen for the testing of "Single level” scenario and also to verify how would the MVN software will manage four simultaneous contact points, at the hands and feet level, with an incompressible surface.
This exercise will not be executed using props, or aiding devices;

"Squats" on the training mattress - was chosen for the testing of "Soft floor" scenario and also to verify how would the MVN software will manage two simultaneous contact points, at the feet level, with a compressible surface. This exercise will be executed using a high-density training mattress, like the type used in Judo;

„Stairs climbing” - was chosen for the testing of „Multi level” scenario and also to verify how would the MVN software will manage two simultaneous contact points, at the feet level, with an incompressible, multilevel-surface. This exercise will be executed using the stepper as an aiding device, to simulate a stair climbing action;

„Balance” - was chosen for the testing of „No level" scenario and also to verify how would the MVN software will manage two simultaneous contact points, at the feet level, with an incompressible surface. This exercise will be executed using the „balance board" as an aiding device, so that even if the feet of the human subject under analysis, are moving on the vertical, his pelvic area should stay at the same level all the time.

For each type of exercise, five analysis sessions were performed. Out of each session, the most representative three were then chosen and in the last phase, after a thorough analysis, only one session was elected, considered to be representative for the exercise in question. A set of five sessions, were performed each day, for each exercise, inserting a day break after each day of analysis, alternating the type of exercise, between the once that are especially targeting the lower limbs and the once that are especially targeting the upper limbs, so that fatigue does not affect the results of the analysis. In all the analysis, the duration of each session was determined in relation to the level of physical stress (the degree of fatigue) imposed by the exercise, this being only an indicative factor, the sessions consisting of a predetermined number of repetitions, or of a predetermined period of time.

On each elected session, only that set of consecutive frames considered to be representative for the exercise was used, i.e. the beginning and the end set of frames were removed, because in those frames, the human subject under analysis was preparing for the start of the exercise, adapting to it, or was completed the exercise, and/or because of the physical stress involved, he was getting out of balance.

The analysis of the images was done by relating the positions of the virtual dummy's body segments to the lines and columns that make up the grid of the virtual reference plane, to the position of virtual reference marker, of the calibration's origin and to the global coordinate system of the MVN software (see Fig. 1). 


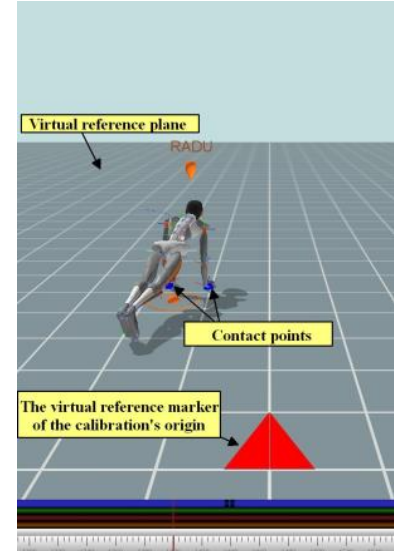

Figure 1: The graphic reference elements of the analysis

The intersection between the baseline of the virtual reference marker, of the calibration's origin and the perpendicular on this line, it's considered the origin of the calibration/analysis. The first grid line, on the virtual reference plane, which contains the virtual reference marker, is considered the upper line number one, and the one under the virtual reference marker is considered the bottom-line number one, in the analysis. The upper lines are in the positive direction of the $0 \mathrm{X}$ axis, and the lower lines in the negative direction of the same axis.

The first column containing the right half of the virtual reference marker is considered the right column number one and the first column containing the left half of the virtual reference marker, it's considered the left column number one. The columns situated to the left of the virtual reference marker are in the positive direction of the axis $0 \mathrm{Y}$ and the ones on the right in the negative direction of this axis. In the Y0Z plane the points above the virtual reference plan are considered positive and the ones below are considered negative, obtained after postprocessing of the measurement data, using the "REPROCESS" function provided by the Xsens MVN software

In all the figures chosen for this paper, are obtained before and after post-processing of the measurement data, using the "REPROCESS" function provided by the Xsens MVN software. In the previous mentioned figures, the blue dots represent the points of contact that materialize the interaction between human subject and the physical elements from his surroundings (see Fig.1). These are in fact support points, that are necessary in order to achieve the imposed movement (e.g.: contact of the hands and toes of the feet with the ground during „Push-ups”).

Elements of interest in all the analyzed session:

the ability of MVN to maintain throughout the entire session, the virtual dummy's position in relation to its global positioning system / virtual reference marker's position, as well as with the virtual reference plan; the ability of MVN to display/maintain, throughout the entire session, all the real world contact points, between the human subject under analysis and his surroundings (the floor, the training mattress, the stepper's pedals, the handles of the parallel bars assembly, or the balance board support surface) at the level of his feet and/or of his hands, depending on the chosen scenario.

\section{The Chosen Analysis Sessions (Exercises) and the Resulting Conclusions}

First of the chosen exercises it's called the „Pushups" on the hard floor and it's used for the testing of "Single level" scenario. In this analysis the virtual reference plan should be located at the floor level, and the contact points should be found at the virtual dummy's hands and toes level.
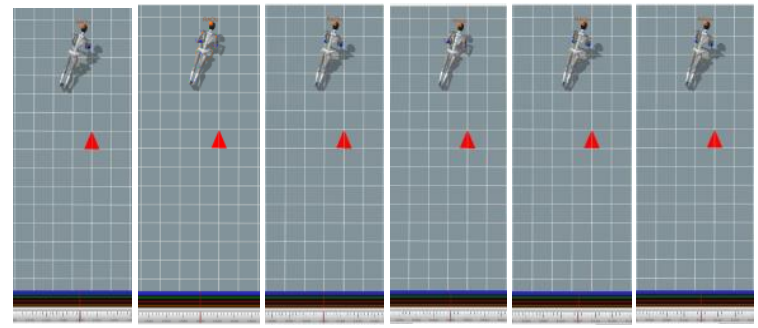

Figure 2: The most graphical suggestive frames for the „Push-ups” exercise, representing the transversecranial plane of the virtual dummy, after the calling of "REPROCESS" function

By comparing the frames in Fig.2, one can observe a "sliding" of the human subject's left hand towards the torso's exterior, during the flexion stages and return to the starting position, during the hand extension stages. This statement is supported by the results presented in charts of Fig.3, Fig.4 and Fig. 7.

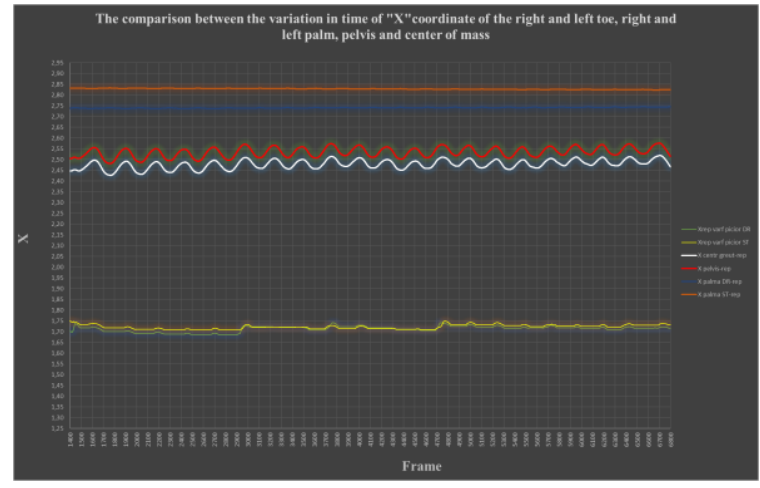

Figure 3: The variations in time of the toes, hands, center of mass and pelvis position's " $X$ " coordinate, of the human subject under analysis, before and after the calling of "REPROCESS" function 
Study on the Influence of Contact Points, Scenarios and Graphical Reference Elements on the Motion Analysis Process, Carried Out Using the Inertial Mechatronic System MVN Analyze

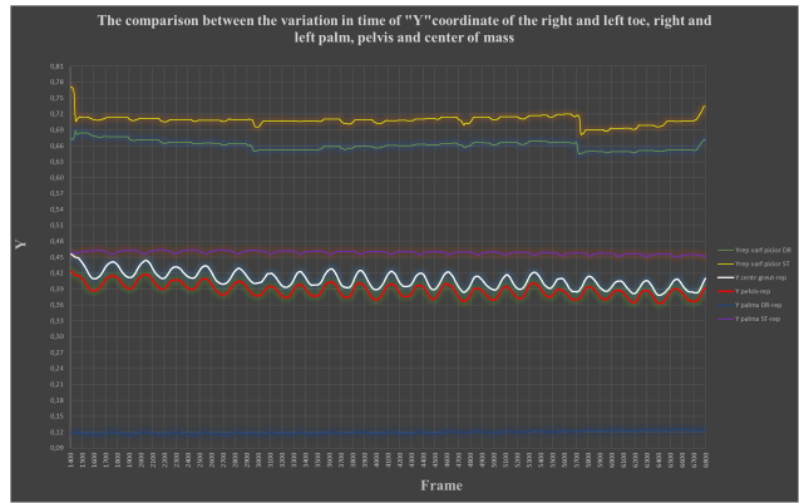

Figure 4: The variations in time of the toes, hands, center of mass and pelvis position's " $Y$ " coordinate, of the human subject under analysis, before and after the calling of "REPROCESS" function

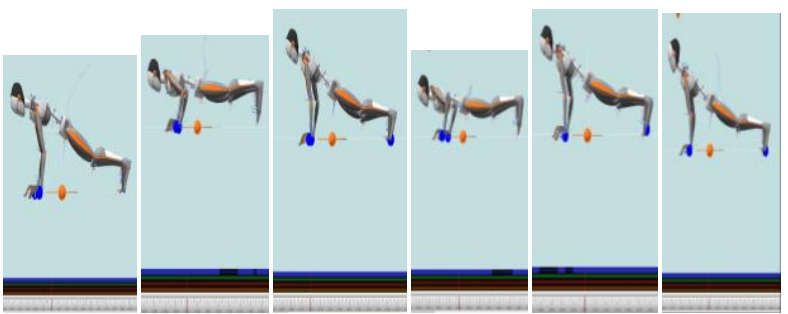

Figure 5: The most graphical suggestive frames for the "Push-ups" exercise, representing the left sagittal plane of the virtual dummy, after the calling of "REPROCESS" function

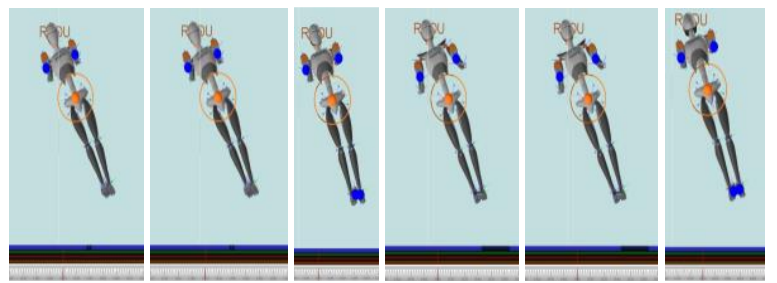

Figure 6: The most graphical suggestive frames for the „Push-ups" exercise, representing the transversecaudal plane of the virtual dummy, after the calling of "REPROCESS" function

Analyzing the views of Fig. 5 and Fig. 6, one can observe a very important aspect:

- although the contact between the toes and hands of the human subject under analysis are pressed against the floor, the contact points are transferring not only between the virtual dummy upper and lower limb, but also between his left and right toe, or between his left and right hand.

This statement is also confirmed by the analysis of the charts presented in the Fig. 3, Fig. 4 and Fig. 7, in which one can see that not only the tose and hands of the virtual dummy are not at the same level, but that also there is a difference of Hight between the left and right toe, or between his left and right hand.

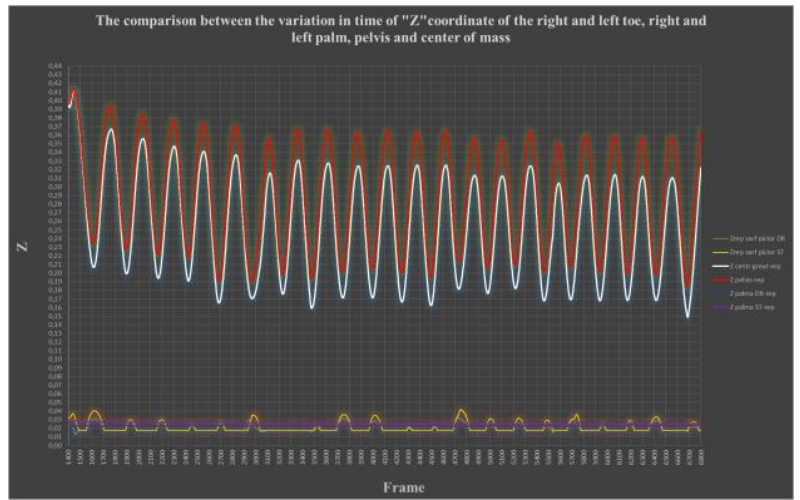

Figure 7: The variations in time of the toes, hands, center of mass and pelvis position's " $Z$ " coordinate, of the human subject under analysis, before and after the calling of "REPROCESS" function

Next chosen exercise it's called „Squats” on the training mattress and it's used for the testing of „Soft floor" scenario. In this analysis the virtual reference plan should be varying in height (floating level), because of the training mattress compressive structure, but this variation is of millimeters order.

The contact points, in this case, should be located at the virtual dummy's toes and heels level.
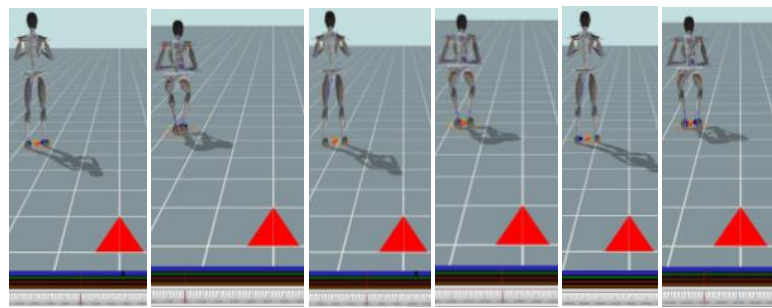

Figure 8: The most graphical suggestive frames for the „Squats” exercise, representing a perspective view, from the back of the virtual dummy, after the calling of "REPROCESS" function

By comparing the frames in Fig.8, one can observe a "sliding" of the human subject's feet towards each other, during the flexion stages and return to the starting position, during the leg extension stages. Another important aspect one can observe is the sliding of the virtual dummy toward his right side.

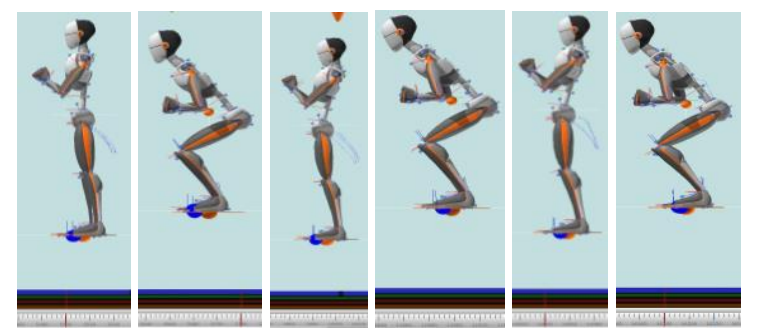

Figure 9: The most graphical suggestive frames for the "Squats" exercise, representing the left sagittal plane of the virtual dummy, after the calling of "REPROCESS" function 
Study on the Influence of Contact Points, Scenarios and Graphical Reference Elements on the Motion Analysis Process, Carried Out Using the Inertial Mechatronic System MVN Analyze

Even though, in the full leg extension stages, the support should be uniformly distributed through the whole surface of each foot's sole, one can observe that MVN's software, it's only placing the contact points, at the virtual dummy's toes level, which is not normal, due to the fact that the training mattress shod be much easier compressed under the heels, in this situation (see Fig. 9).

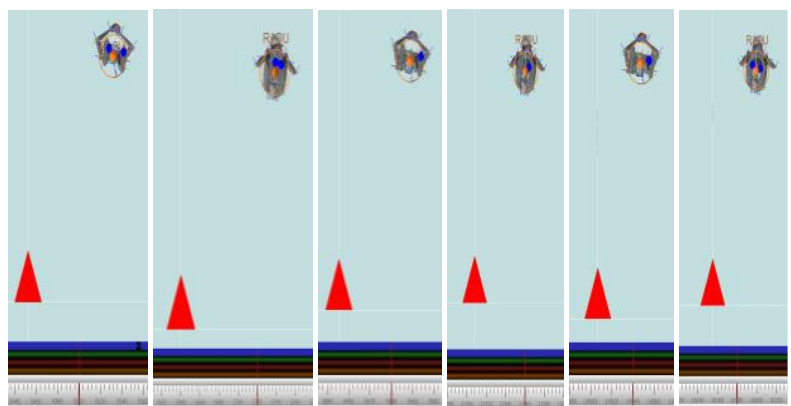

Figure 10: The most graphical suggestive frames for the "Squats" exercise, representing the transversecaudal plane of the virtual dummy, after the calling of "REPROCESS" function

Analyzing the views of Fig. 9 and Fig. 10, one can observe the following facts:

- although the contact between the toes and hands of the human subject under analysis are pressed against the floor, the contact points are transferring not only between the virtual dummy upper and lower limb, but also between his left and right toe.

The above statements are being supported by the results presented in charts of Fig. 11, Fig. 12 and Fig. 13 , in which one can see that there is a difference of Hight between the left and right toe.

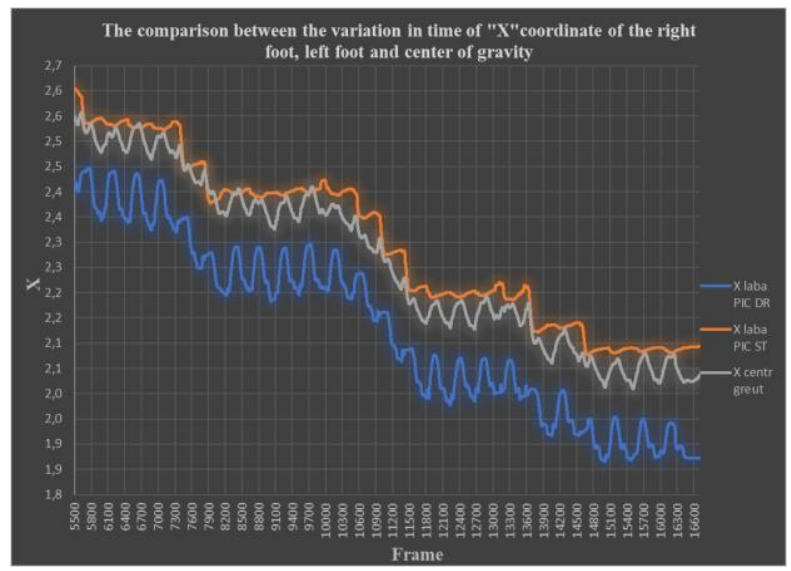

Figure 11: The variations in time of the feet and center of mass position's " $X$ " coordinate, of the human subject under analysis, before and after the calling of "REPROCESS" function

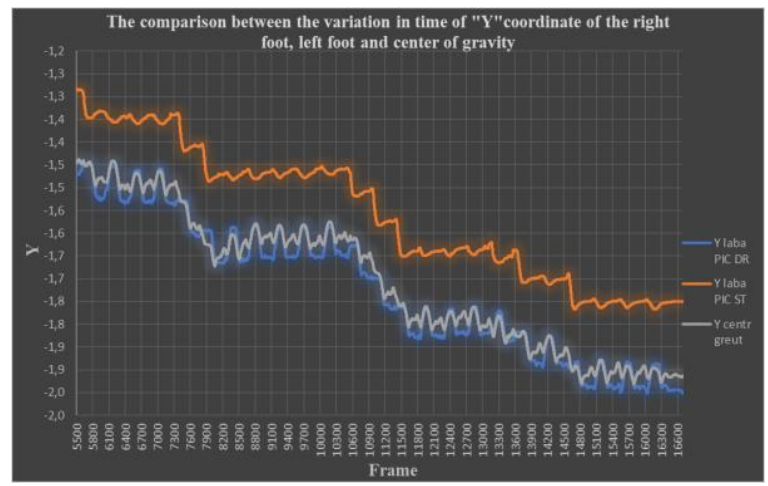

Figure 12: The variations in time of the feet and center of mass position's " $Y$ " coordinate, of the human subject under analysis, before and after the calling of "REPROCESS" function

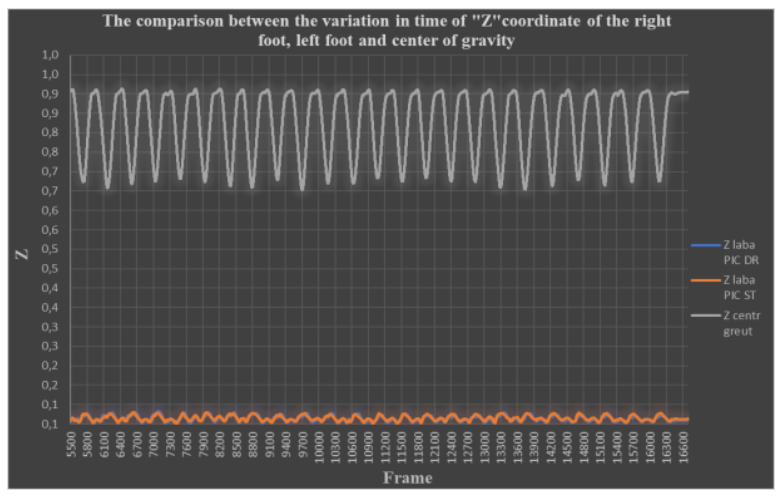

Figure 13: The variations in time of the feet and center of mass position's "Z" coordinate, of the human subject under analysis, before and after the calling of "REPROCESS" function

For the testing of „Multi level” scenario. the chosen exercise it's called "Stairs climbing” and for this, the human subject under analysis will be using a stepper, a device that can simulate the actual action of stairs climbing. In this analysis the virtual reference plan should be varying in height (floating level), due to alternation between the mentioned device's pedals, knowing that while one goes up, the other one goes down. The contact points, in this case, should be located at the virtual dummy's toes level.

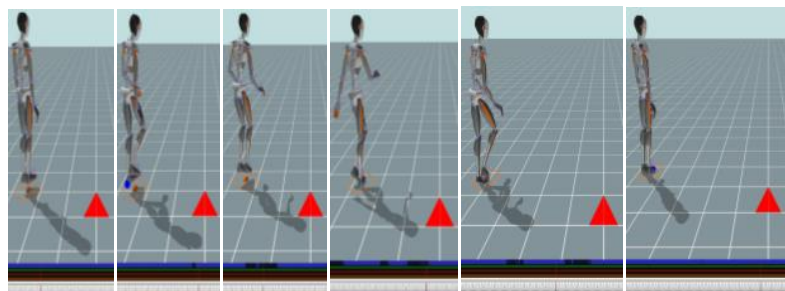

Figure 14: The most graphical suggestive frames for the „Stairs climbing” exercise, representing a perspective view, from the back of the virtual dummy, after the calling of "REPROCESS" function 
Study on the Influence of Contact Points, Scenarios and Graphical Reference Elements on the Motion Analysis Process, Carried Out Using the Inertial Mechatronic System MVN Analyze

By comparing the frames in Fig. 14, one can observe a big "sliding" of the virtual dummy toward his left side.

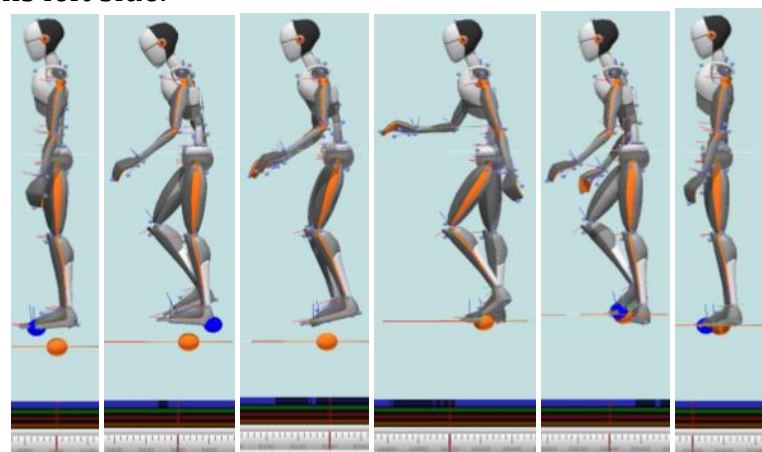

Figure 15: The most graphical suggestive frames for the "Stairs climbing” exercise, representing the left sagittal plane of the virtual dummy, after the calling of "REPROCESS" function

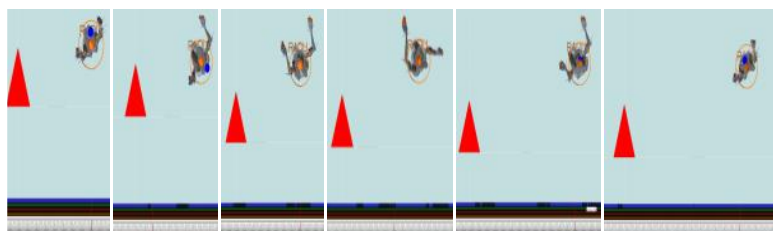

Figure 16: The most graphical suggestive frames for the „Stairs climbing” exercise, representing the transverse-caudal plane of the virtual dummy, after the calling of "REPROCESS" function

Analyzing the views of fig. 15 and fig. 16, one can observe the following facts:

- although the contact between the toes and heels of the human subject under analysis should alternate, meaning that when the left foot is pressing down and the right foot goes up, the contact points should be located at the left toe's and right knee's level and vice versa when the right foot is pressing down and the left foot goes up, the contact points are not placed correctly by the MVN's software.

The above statements are being supported by the results presented in charts of Fig. 17, Fig. 18 and Fig. 19.

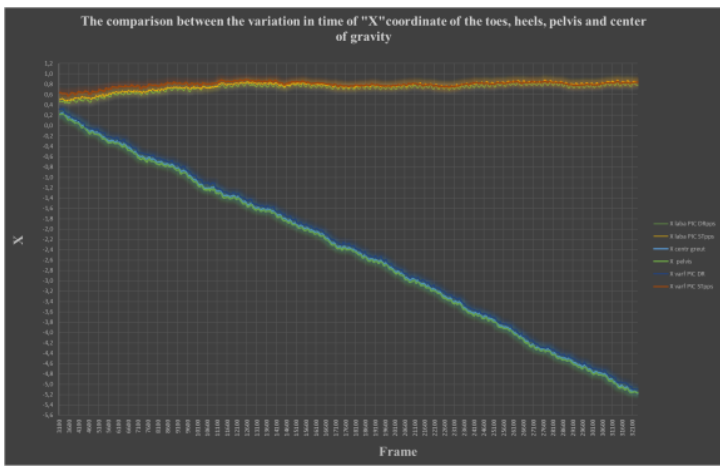

Figure 17: The variations in time of the toes, heels, pelvis and center of mass position's " $X$ " coordinate, of the human subject under analysis, before and after the calling of "REPROCESS" function

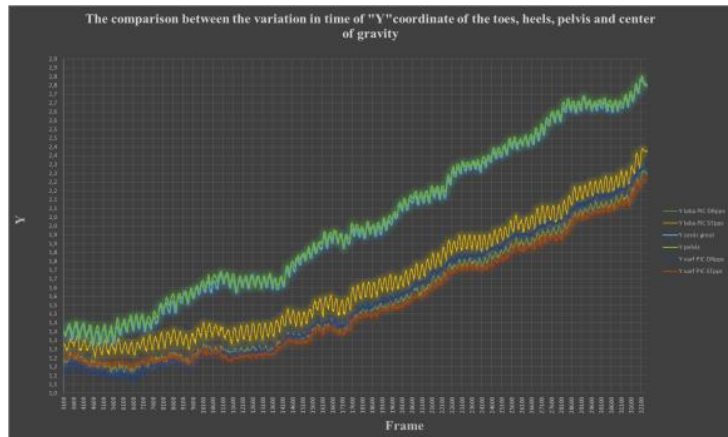

Figure 18: The variations in time of the toes, heels, pelvis and center of mass position's " $Y$ " coordinate, of the human subject under analysis, before and after the calling of "REPROCESS" function

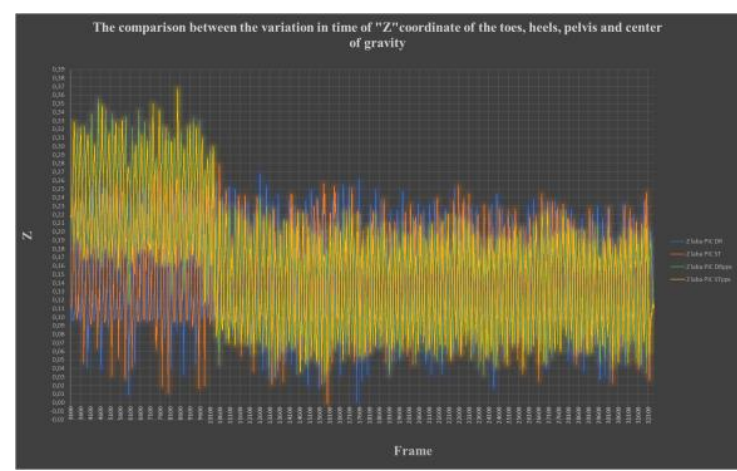

Figure 19: The variations in time of the toes, heels, pelvis and center of mass position's " $Z$ " coordinate, of the human subject under analysis, before and after the calling of "REPROCESS" function

The last of the chosen exercises it's called the „Balance” and it's used for the testing of „No level” scenario. This exercise will be executed using the „balance board" as an aiding device. The pelvic area, of the human subject under analysis, should stay at the same level all the time. In this analysis the virtual reference plan should be located at the upper face of the balance board level, and the contact points should be found at the virtual dummy's feet level.

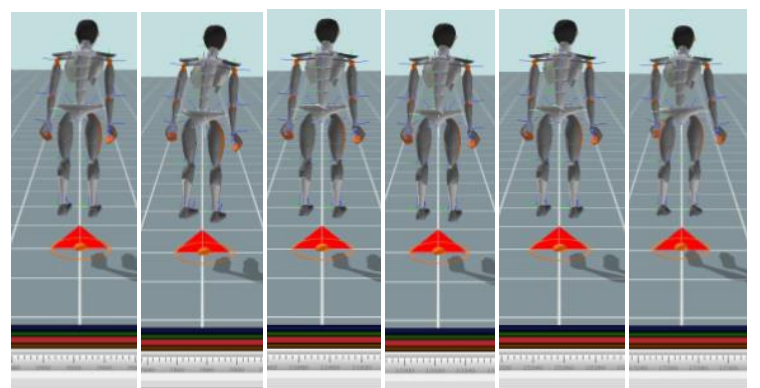

Figure 20: The most graphical suggestive frames for the „Balance” exercise, representing a perspective view, from the back of the virtual dummy, after the calling of "REPROCESS" function

By comparing the frames in Fig.20, one can observe that there are no contact points. 


\section{Conclusions}

The contact points, graphical reference elements and scenarios, are very important tools into the primary, visual, evaluation of the motion analysis results. They are not just a visual aid (used for the visual effect), but they really are a valid indicator of the position and interaction between the human subject, under analysis and the environment into which the action is taking place. Even though, in some situations (e.g.: when the contact points are transferring from the palms to the toes and vice versa, or between right and left palm or toes) those graphical tools can be misleading, this fact might be attributed to the inability of choosing the proper scenario and the right contact level, on the user part.

\section{References}

[1] https://www.xsens.com/

[2] https://www.xsens.com/tags/inertial-sensors/

[3] https://www.gearssports.com/optical-vsinertial-motion-capture/

[4] https://www.sensorsmag.com/components/ove overv-mems-inertial-sensing-technology

[5] "The positioning errors generated by Xsens MVN inertial system during the analysis of <Push-ups $>$ exercise, using the <Single Level> scenario, before and after the calling of <REPROCESS $>$ function", authors: Badea Cristian Radu and Badea Sorin Ionut, IJOMAM-2018, INCDMTM-Bucharest 